\title{
Estresse, ansiedade, depressão e coping materno na anomalia congênita ${ }^{1}$
}

Schwanny Roberta Costa Rambalducci Mofati Vicente. Universidade Federal do Espírito Santo.

Kely Maria Pereira de Paula. Universidade Federal do Espírito Santo.

Franciane Figueiredo da Silva. Universidade de São Paulo.

Camila Nasser Mancini. Universidade Federal do Espírito Santo.

Sarah Almeida Muniz. Centro de Medicina Psicossomática.

\section{Resumo}

O nascimento de um filho com Anomalia Congênita (AC) poderá repercutir na organização familiar, comprometendo aspectos afetivos e a interação da nova díade. Este estudo identificou níveis de estresse, ansiedade e depressão, bem como estratégias de enfrentamento (EE) ou coping adotado por 25 mães de bebês com AC, internados em três UTIN da região; sendo aplicados inventários de estresse (ISSL), ansiedade (BAI) e depressão (BDI), além de entrevista para identificação das EE. A maioria $(n=12)$ estava na fase de resistência do estresse, com nível moderado de ansiedade $(n=8)$, e níveis leve $(n=7)$ e moderado $(n=7)$ de depressão. As mães relataram, nesse período, o uso de EE adaptativas positivas como Autoconfiança e Busca de Suporte, e de EE negativas como Delegação e Desamparo, estas associadas à depressão. Sugere-se a adoção de intervenções dirigidas a essa população a fim de reduzir o impacto gerado pela condição do bebê e sua hospitalização.

Palavras-chave: estresse; ansiedade; depressão; coping; malformação congênita.

Abstract

Maternal stress, anxiety, depression and coping in congenital anomaly. The birth of a child with a congenital anomaly (CA) may have an impact on the familiar organization, disturbing affective aspects and the new dyad interaction. This study identified levels of stress, anxiety, depression, as well as the coping adopted by 25 mothers of babies with CA, admitted in three regional NICU. It was applied inventories for stress (ISSL), for anxiety (BAI) and for depression (BDI) and interview to identify coping strategies. Most of the sample $(n=12)$ was in the resistance level of stress, with moderate level of anxiety $(n=8)$, and mild $(n=7)$ and moderate $(n=7)$ levels of depression. Mothers reported, during this period, the use of positive adaptive coping strategies like Self-reliance and Support Seeking, and negative coping strategies like Delegation and Helplessness, which are associated with depression. It is suggested the adoption of interventions targeted to these mothers in order to reduce the impact created by the babies' condition and by their hospitalization.

Keywords: stress; anxiety; depression; coping; malformations.

\section{Resumen}

El estrés, la ansiedad, la depresión y el coping materno en anomalía congénita. El nacimiento de un hijo con anomalía congénita (AC) puede reverberar en la organización familiar, comprometiendo aspectos afectivos y la interacción de la nueva díada. Este estudio identificó niveles de estrés, ansiedad y depresión, así como las estrategias de afrontamiento (EA) o coping adoptado por 25 madres de bebés con AC, hospitalizados en tres UCIN en la región; siendo aplicado inventarios de estrés (ISSL), ansiedad (BAI) y depresión (BDI), además de entrevista para identificar las EA. La mayoría $(n=12)$ estaban en la etapa de la resistencia del estrés, con niveles moderados de ansiedad $(n=8)$, e niveles leve $(n=7)$ y moderado $(n=7)$ de depresión. Las madres reportaron, en este período, el uso de EA positivas como Confianza en sí Mismo y Búsqueda de Apoyo, y EA negativas como Delegación y Desamparo, estas asociadas con la depresión. Se sugiere la adopción de intervenciones dirigidas a esta población para reducir el impacto emocional negativo generada por el estado del bebé y la hospitalización.

Palabras clave: estrés; ansiedad; depresión; coping; malformación congénita. 
A anomalia congênita (AC) é importante causa de mortalidade em recém-nascidos, caracterizada por estados patológicos originados de fatores causais ou qualquer tipo de alteração durante o desenvolvimento embrionário (World Health Organization [WHO], 2016). Entre 2-5\% dos bebês nascidos com AC, as causas mais comuns são as do sistema nervoso central ou aparelho circulatório (Gili et al., 2015; Guardiola et al., 2009). O nascimento de um bebê com AC poderá influenciar significativamente o estado emocional materno, potencializando dificuldades decorrentes da gestação (Vasconcelos \& Petean, 2009), por apresentar maior investimento afetivo e um novo equilíbrio alostático ${ }^{2}$ (Sousa, Silva, \& Galvão-Coelho, 2015). Sendo assim, é possível que o diagnóstico repercuta de forma negativa no bem estar materno, elevando os níveis de estresse (Barbosa \& Oliveira, 2008), o que intensifica transtornos psicológicos associados.

Pesquisas com pais de crianças com deficiência, incluindo AC (Barbosa \& Oliveira, 2008), têm indicado algum nível de estresse nas populações pesquisadas. $O$ estresse é definido como um estado de tensão que causa ruptura no equilíbrio interno do indivíduo (Lipp, 2000). Portanto, o estresse materno é fator que merece atenção, pois influencia o desenvolvimento do filho (Cousino \& Hazen, 2013), gerando problemas comportamentais e emocionais em crianças e adolescentes (Correia \& Linhares, 2007).

Outro aspecto importante a ser analisado, por estar relacionado às instabilidades emocionais maternas, é a ansiedade, definida como um estado desagradável que gera excitação e sentimento de ameaça (Correia $\&$ Linhares, 2007), constructo que envolve aspectos psicológicos, fisiológicos e sociofamiliares (Gorenstein \& Andrade, 1998). Estudo de Perosa, Silveira e Canavez (2008) indica que maior convívio e familiaridade da mãe com o bebê, condição socioeconômica e apoio de um companheiro são fatores capazes de influenciar os níveis de ansiedade e depressão.

Outro elemento investigado quando se considera o impacto emocional parental é a depressão, associada aos estados de estresse e ansiedade no período gestacional ou após o nascimento do bebê (Correia \& Linhares, 2007). A depressão também pode ser influenciada por aspectos genéticos, psicológicos, culturais ou por mudanças fisiológicas no ciclo vital, sendo um problema cada vez mais comum, sobretudo no período gestacional (Cummings \& Kouros, 2009; Elgar, McGrath, Waschbusch, Stewart, \& Curtis, 2004). É caracterizada principalmente por dificuldade de realizar atividades que eram consideradas agradáveis e, ainda, pela incapacidade em lidar com novas situações (Frizzo \& Piccinini, 2005). Diversos estudos acerca da depressão parental (Moraes et al., 2006; Padovani, Linhares, Carvalho, Duarte, \& Martinez, 2004; Perosa et al., 2008; Perosa, Canavez, Silveria, Padovani, \& Peracoli, 2009) têm apontado índices alarmantes de depressão em cuidadores.

O nascimento de um bebê com diagnóstico de malformação pode influenciar as expectativas em relação ao cuidado materno e acarretar mudanças na estrutura familiar, uma vez que as famílias idealizam, desde a gestação, uma criança perfeita (Perosa et al., 2009), porém, vivenciam a situação de maneira particular (Vasconcelos \& Petean, 2009). Mediante a possibilidade de forte impacto emocional, e considerando que as dificuldades podem ser intensificadas após o diagnóstico, a equipe de saúde deve ser capacitada para auxiliar a mãe nesse momento (Perosa et al., 2008). Nesse sentido, a expectativa não correspondida pode significar uma situação de adversidade para a adaptação do organismo e os níveis de estresse, ansiedade e depressão podem se intensificar. Quando tal situação ocorre, é necessário que o indivíduo utilize estratégias de enfrentamento (coping) que viabilizem e reequilibrem a estabilidade psicológica.

As definições de enfrentamento incluem os esforços voltados para o gerenciamento de eventos estressantes (Grassi-Oliveira, Daruy-Filho, \& Brietzke, 2008). Desse modo, não se deve considerar uma estratégia em si melhor que outra: o que irá determinar seu valor adaptativo será o efeito gerado em longo prazo (Faria \& Seidl, 2005; 2006; Gobatto \& Araujo, 2010).

O coping é estudado em casos individuais e é influenciado por recursos pessoais, mas devemos também considerar o enfrentamento como evento social. Ambientes sociais estão envolvidos no processo de coping, seja como forma de suporte ao enfrentamento ou como fonte de eventos estressantes. Trata-se de um processo dinâmico em que o indivíduo enfrenta de diversas formas um ou vários estressores e, em um mesmo episódio, as formas de enfrentamento podem ser alteradas ao longo da situação (Skinner \& Wellborn, 1994).

Em uma perspectiva desenvolvimentista do coping, aspectos neurofisiológicos são considerados no processo de regulação inicial, que se manifestam por meio de esquemas de ação coordenada presentes desde o nascimento (Skinner \& Zimmer-Gembeck, 2007). Inicialmente, o coping compreende as ações 
reflexas, que dão lugar aos esquemas e viabilizam a reorganização do bebê; mais tarde, por volta dos 2 anos, as crianças começam a apresentar as primeiras repostas voluntárias de enfrentamento (Motta et al., 2015). Assim, este constructo envolve a regulação coordenada entre idade, orientação, coordenação, motivação, modulação, direcionamento do comportamento e emoção, sob condição de estresse (Zimmer-Gembeck, \& Skinner, 2009). Alguns estudos irão investigar as relações entre os processos regulatórios iniciais e a forma de responder aos estressores ao longo do ciclo vital (Compas, 2009; Linhares \& Martins, 2015).

A Teoria Motivacional do Coping - TMC, desenvolvida por Skinner e Wellborn (1994), e ampliada por Skinner, Edge, Altman e Sherwood (2003), conceitua coping como ação regulatória, ou seja, padrões organizados de comportamento, emoção, cognições, ambiente e respostas fisiológicas (Zimmer-Gembeck \& Skinner, 2009). A TMC analisa o constructo a partir da postulação de 12 famílias de coping, que são consideradas como categorias de ordem superior, são elas: Autoconfiança, Busca de suporte, Resolução de problemas, Busca de informação, Acomodação, Negociação, Delegação, Isolamento, Desamparo, Fuga, Submissão e Oposição. As seis primeiras famílias são consideradas adaptativas positivas e as seis últimas adaptativas negativas, assim, todas as famílias são adaptativas de forma imediata, mas em longo prazo o uso constante destas em detrimento daquelas poderá comprometer o desenvolvimento do indivíduo. É uma abordagem teórica recente (Ramos, Enumo, \& Paula, 2015), com poucas pesquisas empíricas que adotam esta perspectiva na compreensão do enfrentamento (Cravinho \& Cunha, 2015; Motta \& Enumo, 2010; Motta et al., 2015; Ramos, 2012).

Nessa nova perspectiva, foi proposto um modelo hierárquico para analisar e mensurar o coping (Skinner et al., 2003; Skinner \& Wellborn, 1994). No nível inferior dessa estrutura encontram-se as instâncias ou comportamentos de coping (o que a pessoa faz ou pensa para lidar com o evento) que podem ser capturados por meio do autorrelato; o segundo nível é composto pelas estratégias de coping que são categorizações das instâncias de coping (considerando a função que cada comportamento exerce); e finalmente, nos níveis mais elevados estão as famílias de coping, conjunto de classificações das estratégias de enfrentamento, com múltiplas funções nos processos adaptativos, que oferecem maior ou menor domínio dos diferentes estressores (Skinner et al., 2003). Tal forma de hierarquização não se traduz em uma avaliação de valor das estratégias como sendo mais ou menos maduras, mas na compreensão das diversas ações e suas respectivas funções nos processos adaptativos.

Estudos sobre coping materno são necessários ao passo que minimizam os impactos que eventos adversos podem ter ao longo do desenvolvimento infantil. Assim, Vasconcelos e Petean (2009) identificaram os modos de enfrentamento e os indicadores afetivos (ansiedade e depressão na fase pré-natal) de gestantes que receberam a notícia de malformação do feto. A despeito da ausência de indicadores clínicos para depressão, a maioria das mães apresentou alto nível de ansiedade. Em relação aos resultados de coping, as autoras identificaram a busca de prática religiosa como a estratégia de enfrentamento mais utilizada pelas participantes.

Conforme foi constatado em demais estudos (Padovani et al., 2004; Perosa et al., 2009), uma situação de adversidade poderá refletir na instabilidade emocional parental implicando em elevados níveis de estresse, ansiedade e depressão, e tal fato exigirá que os pais utilizem diferentes estratégias para lidar com estes eventos. O diagnóstico de algum tipo de $\mathrm{AC}$ em um bebê é considerado um evento adverso, uma vez que, em grande parte das vezes, acarretará em internação em Unidade de Terapia Intensiva Neonatal (UTIN), alteração na rotina e dinâmica familiar, entre outros aspectos, o que consequentemente irá influenciar nas atitudes e sentimentos parentais (Ribas-Junior, Moura, \& Bornstein, 2007). É possível que o número de variáveis negativas aumente, exercendo influência ainda mais intensa no desenvolvimento da criança, dado o elevado nível de estresse materno (Cousino \& Hazen, 2013; Pinto, Padovani, \& Linhares, 2009). Como ainda são poucos os estudos no campo da Psicologia que investigam a influência de variáveis psicológicas maternas no desenvolvimento da criança com malformação, são relevantes as propostas que têm por objetivo avaliar os níveis de estresse, ansiedade e depressão, bem como o coping materno diante do diagnóstico de AC do filho.

\section{Método}

Este estudo descritivo, com delineamento misto, foi realizado em três hospitais de níveis estadual e federal da região em que foi desenvolvida a pesquisa. Principais variáveis investigadas: estresse como variável independente, quantitativa; ansiedade e depressão, como variáveis dependentes, quantitativas; e coping 
sendo variável mediadora, classificada como qualitativa para categorias de enfrentamento e quantitativa para total de estratégias utilizadas (Meltzof, 2011).

Mediante assinatura do Termo de Consentimento Livre e Esclarecido participaram 25 mães de bebês nascidos com AC, internados nas UTIN dos referidos hospitais. A amostra foi composta somente por mães, visto que no hospital do estudo estas eram as principais acompanhantes dos recém-nascidos. Dado o diagnóstico, esses bebês apresentavam comprometimento físico e/ ou intelectual, em geral, submetidos a procedimentos médicos e terapêuticos recorrentes. O diagnóstico dos bebês incluía malformação do aparelho circulatório, sistema nervoso, aparelho digestivo, sistema osteomuscular, anomalias cromossômicas não classificadas em outra parte, malformação congênita dos órgãos genitais e malformações do aparelho urinário. O critério de exclusão para a composição da amostra foi o diagnóstico de distúrbio psiquiátrico materno, e nenhuma participante se enquadrou nessa categoria. Tal critério foi adotado para compor um grupo menos heterogêneo e investigar melhor algumas condições emocionais eliciadas pelos estressores especificados. 0 estudo foi aprovado pelo Comitê de Ética em Pesquisa local (Protocolo $\mathrm{n}^{\circ}$ 03246012.4.0000.5542), em conformidade com a Resolução $196 / 96$ no período de julho a outubro de 2012.

$\mathrm{O}$ estudo foi realizado em duas etapas. $\mathrm{Na}$ primeira, foram utilizados as Escalas Beck de Ansiedade e Depressão (BAI e BDI) (Cunha, 2001), o Inventário de Sintomas de Stress para Adultos (ISSL) (Lipp, 2000), além de protocolo para levantamento de dados sociodemográficos da família, incluindo o Critério de Classificação Econômica Brasil - CCEB (Associação Brasileira de Empresas de Pesquisa [ABEP], 2011). Informações sobre o diagnóstico e tratamento da criança foram levantadas nos prontuários médicos. A primeira etapa da pesquisa foi realizada em um dia, assim como a segunda etapa, que ocorreu no menor intervalo de tempo possível em relação à primeira, com intervalo mínimo de um dia e máximo de 22 dias ( $M=5,72$ dias), considerando a nova rotina das participantes. Nesta, foi aplicada a Entrevista de Coping com base na TMC (Skinner \& Wellborn, 1994; Skinner et al., 2003) e no estudo de Ramos (2012). O instrumento apresenta três perguntas introdutórias sendo uma relacionada às expectativas maternas acerca do desenvolvimento do bebê (ex.: "Como você percebe o desenvolvimento do seu filho?") e duas relacionadas às percepções sobre a situação (ex.: "Na sua opinião, porque acha que isso aconteceu com você?" e "Como você se sente passando por esta experiência?"), 31 questões distribuídas nas 12 famílias de enfrentamento (ex.: Busca de suporte "Você compartilha seus sentimentos com outras pessoas?"; Desamparo "Diante destas situações difíceis você se recusa a acreditar nos acontecimentos?") e uma questão geral de encerramento da entrevista (ex.: "Você deseja acrescentar algo sobre o que conversamos?").

Os instrumentos padronizados foram analisados conforme os critérios normativos. Com o auxílio do software Statistical Package for the Social Sciences - SPSS 18, foram aplicados três testes: Qui-Quadrado, para associação de variáveis qualitativas, além de aplicada uma análise de resíduos ajustados para associação local entre as categorias; Mann Whitney, para comparação de medianas das variáveis quantitativas e qualitativas, para as variáveis qualitativas que possuem mais de dois níveis, foi utilizado o Kruskal-Wallis; e correlação de Spearman, para correlação entre variáveis quantitativas. O nível de significância utilizado foi de $5 \%$.

No tocante ao processamento dos dados da entrevista, os relatos foram classificados a partir das 12 categorias de ordem superior postuladas pela TMC, considerando a função do comportamento no processo adaptativo, avaliada mediante o relato da mãe ao longo da entrevista (Skinner et al., 2003). Estas categorias de ordem superior foram categorizadas a partir dos contextos específicos de enfrentamentos descritos pela amostra. Por exemplo, no Momento do Diagnóstico, quando a mãe buscou compreender o que era a malformação ou prognóstico da doença, relatos que envolviam estas características foram classificados como Busca de Informação. Ainda exemplificando, a mãe adotou Resolução de Problema Quando pensou no que podia fazer pelo bebê ao dizer que ficava atenta ao bebê para identificar algum incomodo ou dor.

\section{Resultados}

\section{Dados Sociodemográficos}

As participantes tinham em média 25 anos (18 - 35 anos) e residiam no estado em que o estudo foi realizado $(n=24)$, sendo a maioria $(n=21)$ multípara. No tocante à escolaridade, 12 participantes completaram o ensino médio e oito mães o ensino fundamental; cinco delas tinham ensino fundamental incompleto e uma não concluiu o ensino médio. 
No que diz respeito ao perfil econômico da amostra $(n=25)$ indicado no CCEB, houve a seguinte distribuição sobre a Renda Média Familiar (RMF): C1 ( $n=9$; RMF de R $\$ 1.459,00)$; C2 ( $n=7$; RMF de R $\$ 962,00)$; B2 ( $n=6$; RMF de R $\$ 2.656,00)$; e D $(n=3$; RMF de R $\$$ $680,00)$. Somente $28 \%$ da amostra tinha algum tipo de ocupação; já todos os pais trabalhavam, sendo este 0 principal provedor financeiro da família.

A análise inferencial (Qui-Quadrado) identificou associação entre variáveis maternas e indicadores emocionais. O fato de a mãe ser primípara esteve associado à ansiedade $(p=0,019)$, com forte associação entre a mãe ser primípara e nível mínimo de ansiedade. No contexto do diagnóstico de AC e hospitalização do filho, as variáveis ser primípara e nível de instrução se associaram a variáveis emocionais, enquanto estresse e ansiedade à depressão (Tabela 1).

\section{Tempo de Diagnóstico e Tempo de Internação}

Em relação ao momento do diagnóstico de AC, a maioria recebeu a notícia antes do nascimento $(n=11)$, algumas ao nascer $(n=7)$ ou, ainda, em dois momentos, antes e após o nascimento $(n=4)$ do bebê, com a confirmação do diagnóstico ou quando outras patologias foram identificadas. Algumas mães receberam a notícia depois do nascimento $(n=3)$. Houve associação entre momento do diagnóstico e uso da categoria Isolamento ( $p=0,012$ ), com forte associação para diagnóstico antes do nascimento com antes e depois do nascimento (Qui-Quadrado). A análise estatística indicou também que algumas variáveis maternas podem influenciar na forma como a situação adversa é vivenciada e enfrentada pela mãe. Relações entre o tempo de internação do bebê e o uso de estratégias adaptativas positivas ( $r=0,511 ; p=0,015)$ foram encontradas. Quanto maior o tempo de internação do bebê maior também o uso de estratégias adaptativas positivas (teste de Spearman), ou seja, o uso destas estratégias esteve correlacionado com a experiência vivenciada.

\section{Estresse, Ansiedade e Depressão}

No que tange à avaliação do estresse, medido pelo ISSL (Lipp, 2000), 84\% da amostra apresentou estresse do tipo psicológico (76\%), nas fases de resistência $(n=12)$,

Tabela 1. Análise de Associação das Variáveis Sociodemográficas e o Uso de Coping pelas Famílias.

\begin{tabular}{|c|c|c|c|c|c|c|c|c|c|c|c|c|c|c|c|c|c|}
\hline & 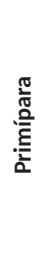 & 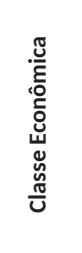 & 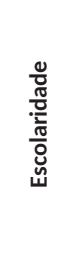 & 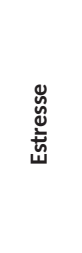 & $\begin{array}{l}\frac{0}{0} \\
\frac{\pi}{0} \\
\frac{d}{n} \\
\frac{\pi}{2}\end{array}$ & 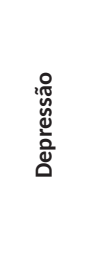 & 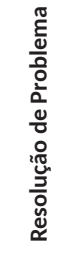 & 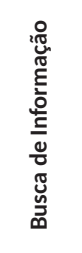 & 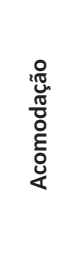 & 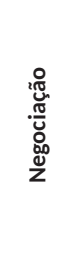 & 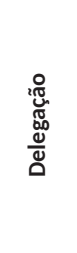 & 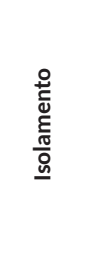 & 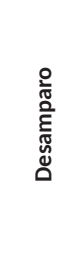 & 兽 & 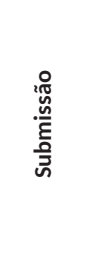 & $\begin{array}{l}\text { 유․ } \\
\text { : } \\
\text { o̊ }\end{array}$ & 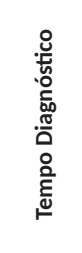 \\
\hline Primípara & - & 0,539 & 0,424 & 0,113 & $0,019^{*}$ & 0,592 & 0,79 & 0,499 & 0,499 & 0,79 & 0,79 & $0,048^{*}$ & 0,334 & 0,312 & $0,048^{*}$ & 0,688 & 0,162 \\
\hline Classe Econômica & - & - & 0,13 & 0,299 & 0,867 & 0,417 & 0,212 & 0,499 & 0,335 & 0,056 & 0,636 & $0,043^{*}$ & 0,176 & 0,394 & 0,306 & 0,773 & 0,097 \\
\hline Escolaridade & - & - & - & 0,39 & 0,323 & $0,046^{*}$ & 0,364 & 0,393 & 0,718 & 0,364 & 0,115 & 0,178 & 0,687 & 0,122 & 0,276 & 0,146 & 0,706 \\
\hline Estresse & - & - & - & - & 0,129 & $0,032^{*}$ & 0,537 & 0,679 & 0,335 & 0,082 & 0,263 & 0,08 & 0,373 & 0,902 & $0,023^{*}$ & 0,149 & 0,762 \\
\hline Ansiedade & - & - & - & - & - & $0,024^{*}$ & 0,112 & 0,393 & 0,499 & 0,462 & 0,987 & 0,626 & 0,176 & 0,426 & 0,155 & $0,001^{*}$ & 0,060 \\
\hline Depressão & - & - & - & - & - & - & 0,607 & 0,448 & 0,448 & 0,094 & 0,607 & 0,309 & 0,135 & 0,051 & $0,029^{*}$ & $0,001^{*}$ & 0,349 \\
\hline $\begin{array}{l}\text { Resolução de } \\
\text { Problema }\end{array}$ & - & - & - & - & - & - & - & 0,55 & 0,55 & 0,61 & 0,216 & 0,959 & 0,237 & $0,032^{*}$ & 0,212 & 0,305 & 0,649 \\
\hline $\begin{array}{l}\text { Busca de } \\
\text { Informação }\end{array}$ & - & - & - & - & - & - & - & - & 0,773 & 0,55 & 0,55 & 0,084 & 0,68 & 0,306 & 0,453 & 0,219 & 0,273 \\
\hline Acomodação & - & - & - & - & - & - & - & - & - & 0,55 & 0,55 & 0,453 & 0,68 & 0,306 & 0,453 & 0,219 & 0,638 \\
\hline Negociação & - & - & - & - & - & - & - & - & - & - & 0,61 & 0,212 & $0,004^{*}$ & 0,659 & $0,016^{*}$ & 0,305 & 0,370 \\
\hline Delegação & - & - & - & - & - & - & - & - & - & - & - & 0,119 & 0,237 & 0,659 & 0,959 & 0,305 & 0,649 \\
\hline Isolamento & - & - & - & - & - & - & - & - & - & - & - & - & 0,404 & 0,569 & 0,103 & 0,91 & $0,012^{*}$ \\
\hline Desamparo & - & - & - & - & - & - & - & - & - & - & - & - & - & 0,766 & $0,012^{*}$ & 0,096 & 0,608 \\
\hline Fuga & - & - & - & - & - & - & - & - & - & - & - & - & - & - & 0,128 & 0,137 & 0,109 \\
\hline Submissão & - & - & - & - & - & - & - & - & - & - & - & - & - & - & - & 0,067 & 0,471 \\
\hline Oposição & - & - & - & - & - & - & - & - & - & - & - & - & - & - & - & - & 0,168 \\
\hline $\begin{array}{l}\text { Tempo } \\
\text { Diagnóstico }\end{array}$ & - & - & - & - & - & - & - & - & - & - & - & - & - & - & - & - & - \\
\hline
\end{tabular}

Nota. Para esta análise estatística foi utilizado o teste Qui-Quadrado. O índice de significância utilizado foi de 5\% 
quase exaustão $(n=6)$, ou mesmo exaustão $(n=3)$. Das mães com níveis de quase exaustão e exaustão $(n=9)$, a maioria recebeu diagnóstico antes do nascimento $(n=6$; quatro antes e duas antes e depois), depois ( $n=$ 2 ) e ao nascer $(n=1)$. Quanto aos níveis de ansiedade, obtidos por meio da Escala Beck, as mães apresentaram este indicador em nível moderado $(n=8)$, níveis mínimo $(n=6)$ e leve $(n=6)$, e cinco participantes em nível grave. Tal como a Escala de Ansiedade, a Escala Beck de Depressão considera os sentimentos da última semana vivida. Os dados mostraram grau mínimo de depressão $(n=8)$, seguido pelos níveis leve $(n=7)$ e moderado ( $n$ $=7$ ), mas três participantes apresentaram depressão em nível mais crítico no instrumento (Figura 1).

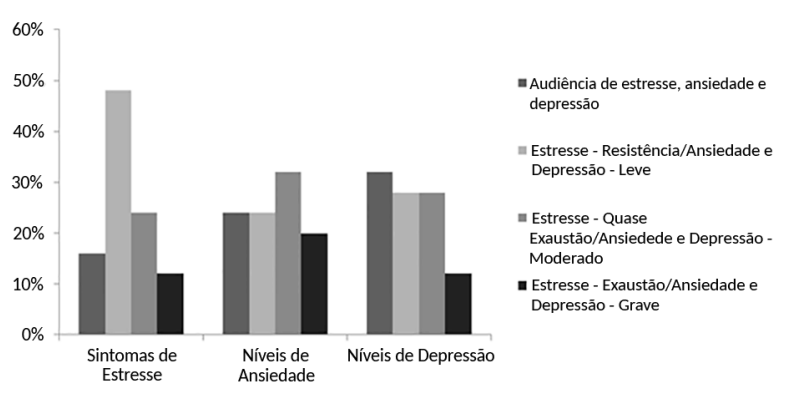

Figura 1. Comparação dos Níveis de Sintomas de Estresse, Ansiedade e Depressão na Amostra $(n=25)$.

Tomando por base a análise inferencial (QuiQuadrado), depressão esteve associada ao grau de escolaridade $(p=0,046)$, a estresse $(p=0,032)$ e ansiedade $(p=0,024)$. Análise dos resíduos ajustados indicou forte associação entre nível mínimo de depressão com ensino fundamental incompleto e nível moderado de depressão com ensino médio incompleto; forte associação entre nível mínimo de depressão com ausência de sintomas de estresse e entre nível leve de depressão com estresse em fase de resistência; forte associação entre nível mínimo de depressão com nível mínimo de ansiedade e entre nível grave de depressão com estresse em fase de exaustão.

\section{Coping Materno}

A entrevista, cujo objetivo foi capturar o enfrentamento materno diante do diagnóstico e da hospitalização do filho, com base na TMC, foi dividida em duas partes. A primeira parte estava relacionada às expectativas maternas acerca do desenvolvimento do bebê e a segunda, composta de perguntas específicas para cada família de coping.
De forma geral, as mães apresentaram boas expectativas acerca do desenvolvimento do bebê (92\%). Metade das respostas $(n=13)$ correspondeu à melhora da criança, ou seja, discorrem sobre a evolução clínica do quadro. Outro aspecto considerado na primeira parte da entrevista foi o motivo da ocorrência da AC, questão também importante para a compreensão a respeito do enfrentamento materno do diagnóstico e da hospitalização do bebê. Parte das respostas estava relacionada ao fato de ter acontecido em função da vontade de Deus (28\%) e problemas ocorridos durante a gestação (28\%). A falta de compreensão sobre o acontecimento apareceu em $24 \%$ das respostas, ou seja, as mães não conseguiam entender o motivo do diagnóstico de $\mathrm{AC}$ ou acreditavam que aconteceu sem motivo específico (12\%). Para uma pequena parcela (4\%) o diagnóstico e a hospitalização foram vistos como forma de aprendizado/crescimento pessoal; e por fim, culpa por algo cometido no passado (4\%).

As famílias de coping, a seguir, serão analisadas, assim como as estratégias de enfrentamento diante do diagnóstico de $A C$ e hospitalização do bebê na UTIN. Adotando-se o modelo da TMC, foi realizada análise do conteúdo das entrevistas, classificadas em categorias adaptativas positivas e negativas (Skinner et al., 2003). Indivíduos que utilizaram ambas as estratégias foram contabilizados nos dois grupos, considerando ou não a utilização daquela estratégia.

A análise descritiva identificou que as mães ( $n$ = 25) utilizaram, de forma geral, os dois grupos de enfrentamento, contudo, houve preponderância no uso de estratégias adaptativas positivas para $64 \%(n=16)$ da amostra. Para $32 \%(n=8)$ houve uso de estratégias tanto adaptativas positivas quanto negativas; e somente uma mãe relatou adotar mais estratégias adaptativas negativas ao lidar com as adversidades.

$\mathrm{Na}$ primeira família de coping investigada, Autoconfiança, utilizada Quando a mãe pensa no que pode fazer pelo bebê (88\%), as estratégias mais frequentemente adotadas foram aceitar responsabilidade e autofala positiva relatadas por $92 \%$ das mães. A segunda família investigada foi Busca de Suporte, mais utilizada Quando a mãe pensa em como se sente (100\%), sendo, busca de conforto e confiança as estratégias utilizadas por toda a amostra. A segunda estratégia mais frequente nesta família foi busca de ajuda, adotada por $92 \%$ da amostra. Na família Resolução de Problemas as estratégias mais utilizadas foram planejar estratégia e encorajamento, efetuadas por $64 \%$ das participantes. Esta família foi mais utilizada pelas participantes Quando 
pensava no que podia fazer pelo bebê (80\%). Busca de Informação, terceira família investigada, foi utilizada predominantemente No momento do diagnóstico (76\%), sendo interesse a estratégia mais utilizada pelas participantes (96\%). Na família Acomodação as estratégias aceitação e convicção foram utilizadas por $88 \%$ das participantes, seguida pela categoria compromisso (72\%); empenho foi utilizado por $56 \%$ da amostra. Esta família foi mais utilizada pela mãe Quando pensava no diagnóstico do bebê (60\%). A sexta família das estratégias adaptativas positivas é a Negociação e, nesta, a estratégia definição de objetivo estava contida nos relatos de 18 mães (72\%). O uso desta família foi identificado Quando a mãe pensava no que podia fazer pelo bebê (60\%).

Na família Delegação, utilizada Quando a mãe pensava no diagnóstico do bebê (52\%), a estratégia depender de outros (76\%) foi a mais identificada nos relatos. Isolamento é a segunda família das estratégias adaptativas negativas e a categoria mais utilizada foi desolação (68\%), seguida de solidão, utilizada por $64 \%$ e afastamento por $52 \%$. Quando a categoria Isolamento foi utilizada, a mãe se referiu à Forma como se sentia (52\%). Desamparo também foi uma categoria utilizada Quando a mãe pensava na forma como se sentia (60\%), e a estratégia dúvidas aparece em grande escala (88\%), seguida por desânimo (80\%), sucumbir (64\%) e confusão (40\%). Fuga foi a categoria menos relatada, sendo identificada Quando a mãe pensava no diagnóstico do bebê (28\%), sendo mais representada por meio das estratégias evitação (56\%), escapar (48\%) e procrastinação (36\%). Para Submissão, identificada Quando a mãe pensava na forma como se sentia (52\%), $76 \%$ das mães utilizaram a estratégia autoculpa, seguida por descontentamento/desgosto (68\%), ruminação (60\%) e pensamentos intrusivos (52\%). A última categoria analisada foi Oposição, pouco referida pela amostra: $32 \%$ utilizaram as estratégias explosão e raiva, identificada de forma predominante Quando a mãe pensava na forma como se sentia (32\%).

Na última pergunta do protocolo, onde as mães se expressavam livremente, apenas oito participantes apresentaram comentários adicionais: a entrevista ajudou a enfrentar a situação vivenciada (56\%); destacaram a fé para superar o diagnóstico e a hospitalização do filho (22\%); ressaltavam que permanecer na UTIN durante o dia ajudava a enfrentar tal situação positivamente (11\%); e reclamações dirigidas aos serviços da UTIN (11\%).

São destacadas as associações (Teste Mann Whitney e Kruskal Wallis) entre as variáveis do estudo - estresse, ansiedade e depressão - e variáveis qualitativas (dados socioedemográficos e famílias de coping) e quantitativas (total de estratégias e tempo de internação do bebê). Os dados indicaram as seguintes associações entre variáveis: a) depressão e uso de estratégias adaptativas negativas ( $p=0,005 ; \mathrm{KW}=12,930) ; \mathrm{b}$ ) Resolução de Problemas e uso de estratégias adaptativas positivas ( $p=0,002 ; \mathrm{KW}=7,500)$; Negociação e uso de estratégias adaptativas positivas $(p=0,002$; KW $=$ $7,500)$, negativas $(p=0,039 ; \mathrm{KW}=15,00)$ e tempo de internação do bebê ( $p=0,014 ; \mathrm{KW}=7,00) ; c)$ Isolamento e uso de estratégias adaptativas negativas $(p=0,049)$; d) Desamparo e uso de estratégias adaptativas positivas $(p=0,02)$ e negativas $(p=0,02)$; e e) Fuga $(p=0,002)$, Submissão $(p=0,001)$ e Oposição $(p=0,001)$, com uso de estratégias adaptativas negativas (Tabela 2).

Análise pelo Qui-Quadrado indicou que a condição da mãe ser primípara esteve associada à ansiedade ( $p$ $=0,019)$ e ao uso das famílias Isolamento $(p=0,048)$ e Submissão $(p=0,048)$, com forte associação mediante a análise de resíduos ajustados; a classe econômica associada ao uso da família Isolamento $(p=0,043)$, com associação entre Isolamento e classe C2; o estresse esteve relacionado ao uso da família Submissão ( $p=$ $0,023)$, associado à fase de resistência do estresse; a ansiedade ao uso de Oposição ( $p=0,001)$, com forte associação de ansiedade em nível mínimo e grave; depressão em nível leve associada ao uso de Submissão ( $p=0,029)$ e Oposição $(p=0,001)$, nível moderado de depressão associado à Oposição; a família Resolução de Problemas associada à Fuga $(p=0,032)$; Negociação ao uso de Desamparo $(p=0,004)$ e Submissão $(p=0,016)$; e, por fim, associação entre Desamparo e Submissão ( $p$ $=0,012$ ) (Tabela 1).

\section{Discussão}

A partir dos resultados foi possível identificar que, na avaliação dos indicadores emocionais, grande parte das mulheres apresentou níveis de estresse (84\%), principalmente na fase de resistência, com predominância de estresse psicológico, ou seja, fase em que sintomas como sensibilidade emotiva excessiva e irritabilidade, por exemplo, estão começando a surgir. Nesta fase, o organismo reage para que não haja o desgaste total de energia (Lipp, 2000). A maioria das mães que apresentou fortes sintomas de estresse (quase exaustão e exaustão) $(n=9)$ recebeu algum diagnóstico do bebê ainda no pré-natal ( $n=6)$, o que indica que, para esta amostra, o momento em que a notícia foi dada 
Coping materno e anomalia congênita

Tabela 2. Comparação entre Medianas pelos Testes de Mann Whitney e Kruskal Wallis* dos Escores da Adaptação Positiva e Negativa e Tempo de Internação, Segundo Variáveis Qualitativas Propostas.

\begin{tabular}{|c|c|c|c|c|c|c|}
\hline & \multicolumn{2}{|c|}{ Adaptação Positiva } & \multicolumn{2}{|c|}{ Adaptação Negativa } & \multicolumn{2}{|c|}{ Tempo de Internação } \\
\hline & Estatística de teste & $p$-valor & Estatística de teste & $p$-valor & Estatística de teste & $p$-valor \\
\hline Classe Econômica* & 1,545 & 0,672 & 2,246 & 0,523 & 3,202 & 0,361 \\
\hline Escolaridade* & 6,014 & 0,111 & 2,693 & 0,441 & 4,918 & 0,178 \\
\hline Estresse* & 2,150 & 0,542 & 5,895 & 0,117 & 4,211 & 0,240 \\
\hline Ansiedade* & 4,250 & 0,236 & 6,181 & 0,103 & 2,419 & 0,490 \\
\hline Depressão* & 3,782 & 0,286 & 12,93 & 0,005 & 3,810 & 0,283 \\
\hline Primípara & 48,500 & 0,903 & 24,000 & 0,068 & 22,000 & 0,233 \\
\hline Busca de Suporte & - & - & - & - & - & - \\
\hline Autoconfiança & - & - & - & - & - & - \\
\hline $\begin{array}{l}\text { Resolução de } \\
\text { Problema }\end{array}$ & 7,500 & 0,002 & 41,00 & 0,939 & 24,500 & 0,327 \\
\hline $\begin{array}{l}\text { Busca de } \\
\text { Informação }\end{array}$ & 4,500 & 0,216 & 9,000 & 0,668 & 8,000 & 0,693 \\
\hline Acomodação & 4,500 & 0,216 & 9,000 & 0,668 & 7,000 & 0,581 \\
\hline Negociação & 7,500 & 0,002 & 15,00 & 0,039 & 7,000 & 0,014 \\
\hline Delegação & 36,00 & 0,597 & 22,00 & 0,126 & 21,000 & 0,473 \\
\hline Isolamento & 43,500 & 0,307 & 27,00 & 0,049 & 40,000 & 0,845 \\
\hline Desamparo & 3,500 & 0,020 & 0,500 & 0,020 & 6,000 & 0,110 \\
\hline Fuga & 54,000 & 0,166 & 21,50 & 0,002 & 57,500 & 0,869 \\
\hline Submissão & 31,500 & 0,054 & 5,500 & 0,001 & 38,000 & 0,461 \\
\hline Oposição & 72,000 & 0,698 & 17,50 & 0,001 & 44,500 & 0,307 \\
\hline
\end{tabular}

Nota. * Para as variáveis que possuíam mais de duas categorias foi utilizado o teste de Kruskal-Wallis (índice de significância utilizado foi de 5\%). - variáveis com apenas uma categoria válida.

pelo profissional de saúde refletiu na experimentação de maior ou menor sintomatologia de estresse. O fato da maioria das mães estar na fase de resistência ao estresse pode ter auxiliado o maior uso de estratégias adaptativas positivas, tais como Autoconfiança, Busca de Suporte e Busca de Informação. É possível que o tempo de hospitalização e diagnóstico de AC do bebê, ainda recente, não tenha produzido reações fisiológicas e comportamentais intensas na amostra (Barbosa \& Oliveira, 2008; Carnier, Rodrigues, \& Padovani, 2012). Por isso, a cronicidade do estresse ao longo do tempo não deverá ser desconsiderada (Pinto et al., 2009). De acordo com Cousino e Hazen (2013), a família inicialmente poderá se mostrar fortalecida frente à doença crônica, no entanto, fatores como a mudança de papéis, as novas responsabilidades (com tratamento médico, por exemplo) e a necessidade de mais recursos financeiros, em função do tratamento, poderão afetar negativamente a dinâmica familiar.

Os resultados desta pesquisa são semelhantes ao estudo realizado por Carnier et al. (2012) com mães de bebês submetidos a procedimentos cirúrgicos, em que a maioria (82\%) da amostra apresentava estresse psicológico, sendo a prevalência na fase de resistência. Pode-se afirmar que cuidadores de crianças diagnosticadas com doenças crônicas, em geral, experimentam níveis mais elevados de estresse (Barbosa \& Oliveira, 2008) se comparados a pais de crianças saudáveis (Cousino \& Hazen, 2013).

Outro aspecto relevante diz respeito ao nível de ansiedade experimentado pelas participantes $(n=19)$, fator que pode estar associado ao nível de depressão. Nos resultados aqui apresentados, o nível mínimo de ansiedade esteve associado ao nível mínimo de depressão. Entretanto, fatores como o diagnóstico de AC, a hospitalização e a possibilidade de acompanhamento médico durante toda a vida geram ansiedade nos familiares e refletem na insegurança em relação ao futuro e bem-estar da criança. O estresse devido ao diagnóstico de malformação e os desafios nos cuidados do bebê poderão potencializar os níveis maternos de ansiedade e depressão (Elgar, et al., 2004; Perosa et al., 2009). Deve- 
se destacar que fatores como suporte social e familiar, sobretudo do companheiro, podem atuar positivamente reduzindo os níveis de depressão e ansiedade maternos (Perosa et al., 2008).

A análise estatística (teste Qui-Quadrado) mostrou associações entre mãe primípara e nível mínimo de ansiedade $(p=0,019)$. Por se tratar de uma amostra em que a maioria contava com o apoio do companheiro, tal fato pode ter contribuído para um menor padrão de ansiedade no grupo (Vasconcelos \& Petean, 2009). Apesar desse suporte, a amostra também adotou estratégias das famílias Isolamento e Submissão, sugerindo dificuldades para lidar com acontecimentos decorrentes do diagnóstico (Skinner et al., 2003). Houve associação entre tempo de diagnóstico e uso da família Isolamento, fortemente ligada ao diagnóstico precoce, indicando que o tempo influencia na forma como a mãe lida com a situação do bebê. A maioria das mães que recebeu o diagnóstico no pré-natal apresentou níveis de estresse, ansiedade e depressão, possivelmente favorecendo a forte associação com o uso de categoria adaptativa negativa. Oposição e Submissão foram categorias utilizadas por mães primíparas pertencentes à classe $C$. Para Perosa e cols. (2008), baixo nível socioeconômico e condições clínicas do bebê são variáveis que podem influenciar indicadores emocionais maternos.

A maioria das participantes apresentou sinais de depressão (68\%), o que colocou o grupo em condição de risco para o desenvolvimento de outros transtornos. Depressão também esteve associada ao uso de estratégias adaptativas negativas. Deve ainda ser considerado o efeito negativo que a depressão materna pode exercer sobre o desenvolvimento da criança (Cummings \& Kouros, 2009). Além da dimensão emocional, essa condição também pode gerar problemas comportamentais (Elgar et al., 2004; Perosa et al., 2009), aumentando os riscos. Outros aspectos, como o diagnóstico em si e a hospitalização, podem elevar os níveis de estresse, ansiedade e depressão, ocasionando o distanciamento da mãe e, consequentemente, influenciar negativamente no curso de vida da criança (Perosa et al., 2008; Pinto et al., 2009), considerando que as mães são as principais estimuladoras do desenvolvimento (Frizzo \& Piccinini, 2005; Vasconcelos \& Petean, 2009).

O grau de escolaridade materno estava associado ao nível de depressão ( $p=0,046$ no Qui-Quadrado). Estudo de Moraes et al. (2006) indicou menor escolaridade e baixo nível socioeconômico relacionados com níveis depressão. De forma distinta, este estudo apresentou que maior grau de instrução se associou a maior nível de depressão. São necessários novos estudos com amostra ampla para confirmar as associações entre essas variáveis.

Ainda associada à variável depressão pode-se verificar o uso das famílias Oposição e Submissão. Essas duas categorias também possuem relação com outras variáveis, sendo Oposição associada à ansiedade nos níveis mínimo e grave e Submissão ao estresse em fase de resistência. Em outras palavras, esses resultados indicam que a predominância de níveis clínicos de estresse, ansiedade e depressão na amostra esteve associada ao uso de estratégias adaptativas negativas como Oposição e Submissão. O teste Mann Whitney para comparação de medianas confirmou esses dados, considerando que depressão esteve associada ao uso de estratégias adaptativas negativas. Cabe ressaltar que estas estratégias não são utilizadas somente por indivíduos que estão sob forte impacto emocional (Skinner et al., 2003). Isto ocorre em decorrência de uma variabilidade comportamental do indivíduo, por exemplo, neste estudo, Resolução de Problemas esteve associada à Fuga. Ao longo do processo de enfrentamento é possível lançar mão de várias estratégias, sejam de famílias adaptativas positivas ou negativas, para lidar com o estressor (Ramos, 2012). O mesmo ocorre com o uso de Oposição, que esteve associada a dois níveis de ansiedade.

Em consonância com resultados do estudo de Perosa et al. (2009), o diagnóstico de AC e as possíveis intercorrências no quadro de saúde do bebê agravaram de forma expressiva os níveis de estresse, ansiedade e depressão maternos. Saviani-Zeoti e Petean (2015) verificaram níveis de ansiedade e depressão significativamente mais altos entre mulheres com gravidez de risco, especialmente em casos de suspeita de malformação do feto, onde as mães apresentaram níveis moderado a grave de ansiedade e depressão.

No que tange ao conhecimento materno sobre desenvolvimento, possivelmente influenciando os próprios sentimentos de apego, o que, por sua vez, implica no desenvolvimento do bebê (Ribas-Junior et al., 2007), a maioria relatou ter sentimentos positivos (crescimento pessoal, se sentir fortalecida, por exemplo). Tais dados são consonantes com os resultados encontrados na análise do coping, em que a maior parte da amostra utilizou mais estratégias adaptativas positivas. Dependendo do momento vivenciado pela mãe, ou mesmo, dos sentimentos diante do acontecimento, as estratégias são utilizadas de modo a viabilizar a melhor 
adaptação à situação. Se o conjunto de estratégias adotadas conduzirá ou não a um desfecho positivo no desenvolvimento, é preciso investigar e compreender o fenômeno do coping como processo, pois mudanças ocorrem ao longo do tempo, assim como as situações experimentadas (Skinner et al., 2003; Zimmer-Gembeck \& Skinner, 2009).

Como referido, as estratégias de enfrentamento foram distribuídas, segundo o modelo da TMC, em adaptativas positivas e negativas. Nesta amostra, um conjunto de estratégias adaptativas positivas foi usado com maior frequência, sobretudo das famílias Autoconfiança e Busca de Suporte. Apesar da distinção conceitual existente entre essas categorias, pode-se afirmar que, de certa maneira, são complementares, uma vez que Autoconfiança diz respeito à maneira que a pessoa encontra para coordenar a confiança e proteger recursos sociais disponíveis e Busca de Suporte se refere à utilização de tais recursos (Skinner et al., 2003). Nesta categoria foi possível identificar estratégias relacionadas à religiosidade, tema que apareceu de forma acentuada no decorrer das entrevistas, mesmo para as mães que diziam não pertencer ou frequentar determinada religião, pois em algum momento fizeram uso de tal recurso. De igual modo, no estudo de Ramos (2012), as mães enfrentaram a prematuridade do filho adotando essas duas famílias de coping em todas as etapas da pesquisa (antes e depois da alta hospitalar).

A religiosidade é inerente a diversas culturas e por tal motivo possibilita o desencadeamento de recursos psicoafetivos que contribuem de maneira significativa para o enfrentamento das adversidades (Faria \& Seidl, 2005; Gobatto \& Araujo, 2010). Toda a amostra relatou muito apego à fé para lidar com a situação do filho, resultado também destacado por Vasconcelos e Petean (2009). Assim, comportamentos relacionados à fé têm contribuído para o enfrentamento de situações críticas que surgem ao longo da vida, além de uma avaliação positiva da situação. Para as mães, todos os acontecimentos associados ao diagnóstico de malformação estavam oferecendo a elas e à família a chance de crescimento espiritual (Pargament, 2011). De acordo com Faria e Seidl (2006), o uso de estratégias de cunho religioso favorece um resultado adaptativo positivo, pois estimulam a integração social e bemestar. Nesse sentido, as estratégias busca de conforto e confiança foram utilizadas de forma unânime na categoria Busca de Suporte, que também abarca a prática religiosa, sendo a fé um elemento muito importante destacado pela amostra.

Diante das análises descritas, pode-se afirmar que os instrumentos utilizados neste estudo, especialmente a entrevista de coping, foram adequados para investigar como o diagnóstico de malformação do bebê afeta a mãe em face aos recursos que adota para lidar com essa nova condição. Por sua temática e modelo teórico adotado, as contribuições desta pesquisa no campo da Psicologia Pediátrica ampliam o conjunto de estudos empíricos no país que investigam o coping a partir da perspectiva motivacional por meio da TMC (Cravinho \& Cunha, 2015; Grassi-Oliveira et al., 2008; Motta \& Enumo, 2010; Motta et al., 2015; Ramos, 2012; Ramos et al., 2015). Ainda, com a avaliação do impacto emocional materno após o nascimento do bebê, e com a investigação das estratégias de enfrentamento utilizadas, é possível organizar programas de prevenção e planejar intervenções precoces, de um lado, minimizando efeitos deletérios no desenvolvimento da criança e, de outro, com base na promoção de saúde, maximizar a qualidade de vida materna (Cousino \& Hazen, 2013) e bem-estar familiar.

\section{Conclusão}

Diante da AC, a equipe de saúde deverá considerar, nos serviços de saúde materno-infantil, quais são os recursos oferecidos às famílias para vivenciar essa nova condição, uma vez que a previsibilidade de intercorrências negativas poderá minimizar o nível de estresse parental. Os indicadores emocionais encontrados na amostra reforçam a importância de programas de intervenção direcionados a essa população, centrados na temática do coping, a fim de ampliar o repertório de estratégias adaptativas positivas acionadas para lidar com a malformação e os estressores associados ao diagnóstico.

Cabe ressaltar que estudos, principalmente de cunho longitudinal, permitirão avaliar com maior amplitude o impacto que a adoção de estratégias de enfrentamento adaptativas negativas terá ao longo do desenvolvimento do bebê com AC. Mais especificamente, novos estudos empíricos, de delineamentos mais controlados, com base na TMC, poderão ampliar a compreensão sobre a forma como as variáveis estresse, ansiedade, depressão e coping parental se interrelacionam dentro de um modelo compreensivo sobre os processos adaptativos. 


\section{Referências}

Associação Brasileira de Empresas de Pesquisa. (2011). Critério de classificação econômica Brasil. Recuperado de http://www.abep. org/novo/Content.aspex?ContentID=301

Barbosa, A. J. G., \& Oliveira, L. D. (2008). Estresse e enfrentamento em pais de pessoas com necessidades especiais. Psicologia em Pesquisa, 2(2), 36-50. Recuperado de http://pepsic.bvsalud.org/ scielo.php?script=sci_arttext $\&$ pid=S1982-12472008000200005\&ln $\mathrm{g}=$ pt\&tlng=en.

Carnier, L. E., Rodrigues, O. M. P. R., \& Padovani, F. H. P. (2012). Stress materno e hospitalização infantil pré-cirúrgica. Estudos de Psicologia, 29(3), 315-325. doi: 10.1590/S0103-166X2012000300002.

Compas, B. E. (2009). Coping, regulation, and development during childhood and adolescence. In E. A. Skinner \& M. J. Zimmer-Gembeck (Orgs.), Coping and the development of regulation: New directions for child and adolescent development (pp. 87-99). San Francisco: Jossey-Bass. doi:10.1002/cd.245

Correia, L. L., \& Linhares, M. B. M. (2007). Ansiedade materna nos períodos pré e pós-natal: Revisão da literatura. Revista Latino-Americana de Enfermagem, 15(4), 175-182. doi: 10.1590/S010411692007000400024

Cousino, M. K., \& Hazen, R. A. (2013). Pareting stress among caregivers of children with chronic illness: A systematic review. Journal of Pediatric Psychology, 38(7), 1-20. doi: 10.1093/jpepsy/jst049.

Cravinho, C. R. M., \& Cunha, A. C. B. (2015). Enfrentamento da morte fetal pela enfermagem na abordagem disposicional e na Teoria Motivacional do Coping. Estudos de Psicologia, 32(2), 307-318. doi: 10.1590/0103-166X2015000200014

Cummings, M., \& Kouros, C. D. (2009). Maternal depression and its relation to children's development and adjustment. Encyclopedia on Early Childhood Development. Recuperado de http://www.child-encyclopedia.com/en-ca/home.html

Cunha, J. A. (2001). Manual da versão em português das Escalas Beck. São Paulo: Casa do Psicólogo

Elgar, F. J., McGrath, P. J., Waschbusch, D. A., Stewart S. H., \& Curtis, L. J. (2004). Mutual influences on maternal depression and child adjustment problems. Clinical Psychology Review, 24, 441-459. doi: 10.1016/j.cpr.2004.02.002

Faria, J. B., \& Seidl, E. M. F. (2005). Religiosidade e enfrentamento em contextos de saúde e doença. Psicologia: Reflexão e Crítica, 18(3), 381-389. doi: 10.1590/S0102-79722005000300012.

Faria, J. B., \& Seidl, E. M. F. (2006). Religiosidade, enfrentamento e bem-estar subjetivo em pessoas vivendo com HIV/AIDS. Psicologia em Estudo, 11(1), 155-164. doi: 10.1590/S1413-73722006000100018

Frizzo, G. B., \& Piccinini, C. (2005). Interação mãe-bebê em contexto de depressão materna: aspectos teóricos e empíricos. Psicologia em Estudo, 10(1), 57-55. doi: 10.1590/S1413-73722005000100007

Gili, J. A., Poletta, F. A., Pawluk, M., Gimenez, L. G., Campaña, H., Castilla, E., \& López-Camelo, J. S. (2015). High birth prevalence rates for congenital anomalies in South American regions. Epidemiology, 26(5), e53-e55 doi:10.1097/EDE.0000000000000345

Gobatto, C. A., \& Araujo, T. C. C. F. (2010). Coping religioso-espiritual: reflexões e perspectiva para a atuação do psicólogo em oncologia. Revista da Sociedade Brasileira de Psicologia Hospitalar, 13(1), $52-$ 63. Recuperado de http://pepsic.bvsalud.org/scielo.php?script=sci_ arttext\&pid=S1516-08582010000100005\&lng=pt\&tlng=pt
Gorenstein, C., \& Andrade, L.H.S.G.(1998). Inventário de depressão de Beck: propriedades psicométricas da versão em português. Revista de Psiquiatria Clínica, 25(5), 245-250.

Grassi-Oliveira, R., Daruy-Filho, L., \& Brietzke, E, (2008). Coping como função executiva. Psico, 39(3), 275-281. Recuperado de http:// revistaseletronicas.pucrs.br/ojs/index.php/revistapsico/article/ view/3852/3368

Guardiola, A., Koltermann, V., Aguiar, P. M., Grossi, S. P., Fleck, V., Pereira, E. C., \& Pellanda, L. (2009). Neurological congenital malformations in a tertiary hospital in south Brazil. Arquivos de Neuro-Psiquiatria, 67(3B), 807-811. doi:10.1590/S0004-282X2009000500005

Linhares, M. B. M., \& Martins, C. B. S. (2015). O processo da autorregulação no desenvolvimento de crianças. Estudos de Psicologia.32(2), 281-293. doi: 10.1590/0103-166X2015000200012

Lipp, M. N. (2000). Manual do inventário de sintomas de stress para adultos de Lipp (ISSL). São Paulo: Casa do Psicólogo.

Meltzof, J. (2011). Critical thinking about research: psychology and related fields ( $2^{\mathrm{a}} \mathrm{ed}$.). Washington, D.C.: American Psychological Association.

Moraes, I. G. S., Pinheiro, R. T., Silva, R. A., Horta, B. L., Sousa, P. L. R., \& Faria, A. D. (2006). Prevalência da depressão pós-parto e fatores associados. Revista de Saúde Pública, 40(1), 65-70. doi: 10.1590/ S0034-89102006000100011

Motta, A. B., \& Enumo, S. R. F. (2010). Intervenção lúdica para o enfrentamento da hospitalização de crianças com câncer. Psicologia: Teoria e Pesquisa, 26(3), 445-454. doi: 10.1590/S0102-37722010000300007.

Motta, A. B., Perosa, G. B., Barros, L., Silveira, K. A., Lima, A. S. da S. Carnier, L. E., ... Caprini, F. R. (2015). Comportamentos de coping no contexto da hospitalização infantil. Estudos de Psicologia, 32(2), 331-342. doi: 10.1590/0103-166X2015000200016

Padovani, F. P., Linhares, M. M., Carvalho, A. V., Duarte, G., \& Martinez, F. E. (2004). Avaliação de sintomas de ansiedade e depressão em mães de neonatos pré-termo durante e após hospitalização em UTI-Neonatal. Revista Brasileira de Psiquiatria, 26(4), 251-254. doi 10.1590/S1516-44462004000400009

Pargament, K. I. (2011). Religion and coping: The current state of knowkedge. In: S. Folkman (Org.), The Oxford handbook of stress, health, and coping ( $1^{\text {a }}$ ed., pp. 269-288). Nova lorque: Oxford University Press.

Perosa, G. B., Canavez, I. C., Silveira, F. P., Padovani, F. P., \& Peracoli, J. (2009). Sintomas depressivos e ansiosos em mães de recém-nascidos com e sem malformações. Revista Brasileira de Ginecologia e Obstetrícia, 31(9), 433-439. doi: 10.1590/S010072032009000900003

Perosa, G. B., Silveira, F. P., \& Canavez, I. (2008). Ansiedade e depressão em mães de recém-nascidos com malformações visíveis. Psicologia: Teoria e Pesquisa, 24(1), 29-36. doi: 10.1590/S010237722008000100004

Pinto, I. D., Padovani, F. H. P., \& Linhares, M. B. M. (2009). Ansiedade e depressão materna e relatos sobre o bebê prematuro. Psicologia: Teoria e Pesquisa, 25(1), 75-83. doi: 10.1590/S0102-37722009000100009

Ramos, F. P. (2012). Grupo de mães na UTIN: promoção de estratégia de enfrentamento maternas (Tese de Doutorado não publicada). Universidade Federal do Espírito Santo, Vitória, ES.

Ramos, F. P., Enumo, S. R. F., \& Paula, K. M. P. (2015). Teoria motivacional do coping: uma proposta desenvolvimentista de análise do enfrentamento do estresse. Estudos de Psicologia, 32(2), 269-279. doi: 10.1590/0103-166X2015000200011 
Ribas-Junior, R. C., Moura, M. L. S., \& Bornstein, M. H. (2007). Cognições maternas acerca da maternidade do desenvolvimento humano: uma contribuição ao estudo da psicologia parental. Revista Brasileira de Crescimento e Desenvolvimento Humano, 17(1), 104-113. doi: doi: 10.7322/jhgd.19819

Saviani-Zeoti, F., \& Petean, E. B. L. (2015). Apego materno-fetal, ansiedade e depressão em gestantes com gravidez normal e de risco: estudo comparativo. Estudos de Psicologia, 32(4), 675-683. doi: 10.1590/0103-166X2015000400010

Skinner, E. A., \& Wellborn, J. G. (1994). Coping during childhood and adolescence: A motivational perspective. In: D. Featherman, R. M. Lerner, \& M. Perlmutter (Orgs.), Life-span development and behavior (pp. 91-133). Hillsdale: Erlbaum.

Skinner, E. A., \& Zimmer-Gembeck, M. J. (2007). The development of coping. Annual Review of Psychology, 58, 119-44. doi:10.1146/ annurev.psych.58.110405.085705
Skinner, E. A., Edge, K., Altman, J., \& Sherwood, H. (2003). Search for the structure of coping: A review and critique of category systems for classifying ways of coping. Psychological Bulletin, 129(2), 216-269. doi: 10.1037/0033-2909.129.2.216

Sousa, M. B. C. de, Silva, H. P. A., \& Galvão-Coelho, N. L. (2015). Resposta ao estresse: I. Homeostase e teoria da alostase. Estudos de Psicologia, 20(1), 1-10. doi:10.5935/1678-4669.20150002

Vasconcelos, L., \& Petean, E. L. (2009). O impacto da malformação fetal: indicadores afetivos e estratégias de enfrentamento em gestantes. Psicologia, Saúde \& Doenças, 10(1), 69-82. Recuperado de <http://www.scielo.gpeari.mctes.pt/scielo.php?script=sci_ arttext\&pid=S1645-00862009000100006\&lng=pt\&nrm=iso > .

World Health Organization. (2016). Congenital anomalies. Recuperado de http://www.who.int/mediacentre/factsheets/fs370/en/

Zimmer-Gembeck, M. J., \& Skinner, E. A. (2009). Coping, developmental influences. In: H. Reis \& S. Sprecher (Orgs.), Encyclopedia of human relationships. Newbury Park: Sage

\footnotetext{
${ }^{1}$ Este trabalho é fruto de parte da dissertação de mestrado da primeira autora, orientado pela segunda autora. Mestrado financiado pela CAPES.

${ }^{2}$ Sistemas responsáveis pela regulação do organismo e que aumentam a capacidade de resposta eficiente diante de determinada demanda.
} 
Schwanny Roberta Costa Rambalducci Mofati Vicente, Mestre em Psicologia pela Universidade Federal do Espírito Santo (UFES), é Doutoranda no Programa de Pós-Graduação em Psicologia da Universidade Federal do Espírito Santo (UFES). Endereço para correspondência: Avenida Anísio Fernandes Coelho, no 1340, apto. 303, Ed. Brunella, Jardim da Penha, Vitória - ES. CEP: 29060-670. Telefones: (27) 9 9740-7559 ou

(27) 3019-1683. E-mail: schwanny.vicente@gmail.com

Kely Maria Pereira de Paula, Doutora em Psicologia pela Universidade Federal do Espírito Santo (UFES), é Professora

Permanente no Departamento de Psicologia Social e do Desenvolvimento (DPSD) da Universidade Federal do Espírito Santo (UFES). E-mail: kelymppaula@gmail.com

Franciane Figueiredo da Silva, Mestre em Saúde Coletiva pela Universidade Federal do Espírito Santo (UFES), é Doutoranda em Epidemiologia na Universidade de São Paulo (USP). E-mail: francianefigueiredo@gmail.com

Camila Nasser Mancini, Psicóloga formada pela Universidade Federal do Espírito Santo (UFES), é Mestranda no Programa de Pós-Graduação em Psicologia da Universidade Federal do Espírito Santo (UFES). E-mail: camilanmancini@gmail.com

Sarah Almeida Muniz, Psicóloga formada pela Universidade Federal do Espírito Santo (UFES), é Psicóloga Clínica no Centro de Medicina Psicossomática (CEMEPS). E-mail: sarinham@hotmail.com 